\title{
The Topology of the Space of Holomorphic Maps with Bounded Multiplicity
}

\author{
By
}

Kohhei Yamaguchi*

\begin{abstract}
For a complex (quasi-) projective variety $X \subseteq \mathbb{C P}^{N}$ with $\pi_{2}(X)=\mathbb{Z}$ and an integer $d \geq 0$, let $\operatorname{Hol}_{d}^{*}\left(S^{2}, X\right)$ denote the space consisting of all basepoint preserving holomorphic maps $f$ from $S^{2}$ to $X$ with degree $d$. We study the topology of certain subspaces of $\operatorname{Hol}_{d}^{*}\left(S^{2}, X\right)$ defined using the concept of multiplicity of roots, and we show that the Atiyah-Jones-Segal type theorem ([1], [11]) holds for these subspaces if $X$ is belong to a certain family of complex quasi-projective varieties.
\end{abstract}

\section{$\S 1$. Introduction}

If $d \geq 0$ is an integer and $X \subseteq \mathbb{C P}^{m-1}$ is a complex (quasi-) projective variety with the condition $\pi_{2}(X)=\mathbb{Z}$, we denote by $\operatorname{Hol}_{d}^{*}\left(S^{2}, X\right)$ the space consisting of all base point preserving holomorphic maps (i.e. algebraic curves) $f: S^{2} \rightarrow X$ with $[f]=d \in \mathbb{Z}=\pi_{2}(X)$. The corresponding space of continuous maps is denoted by $\operatorname{Map}_{d}^{*}\left(S^{2}, X\right)=\Omega_{d}^{2} X$. The principal motivation for this paper derives from the work of G. Segal [11], in which he investigates the topology of the space $\operatorname{Hol}_{d}^{*}\left(S^{2}, X\right)$ for $X=\mathbb{C P}^{m-1}$ as follows.

Theorem $1.1([\mathbf{1 1}])$. Let $m \geq 2$ and $d \geq 1$ be integers. Then the inclusion $i_{d}: \operatorname{Hol}_{d}^{*}\left(S^{2}, \mathbb{C P}^{m-1}\right) \rightarrow \Omega_{d}^{2} \mathbb{C P}^{m-1}$ is a homotopy equivalence up to dimension $(2 m-3) d$.

Communicated by K. Saito. Received May 6, 2003.

2000 Mathematics Subject Classification(s): Primary 55P10; Secondary 55P35, 55P15.

Key words: algebraic curves, holomorphic maps, homotopy equivalence

Partly supported by Grant-in-Aid for Scientific Research (C) 16540056, Japan Society of the Promotion of Science.

* Department of Information Mathematics, University of Electro-Communications, Chofugaoka, Chofu, Tokyo 182-8585, Japan.

e-mail: kohhei@im.uec.ac.jp

(C) 2006 Research Institute for Mathematical Sciences, Kyoto University. All rights reserved. 
Remark. We say that a map $f: X \rightarrow Y$ is a homotopy equivalence (resp. homology equivalence) up to dimension $N$ if the induced homomorphism $f_{*}$ : $\pi_{i}(X) \rightarrow \pi_{i}(Y)$ (resp. $f_{*}: H_{i}(X ; \mathbb{Z}) \rightarrow H_{i}(Y ; \mathbb{Z})$ ) is bijective when $i<N$ and is surjective when $i=N$.

In this paper, we shall investigate whether a similar result hold for certain subspaces of $\operatorname{Hol}_{d}^{*}\left(S^{2}, X\right)$ introduced from the concept of multiplicity.

For a space $X$, we denote by $\mathrm{SP}^{d}(X)$ the $d$-th symmetric product of $X$. By definition, this is the quotient space $X^{d} / \Sigma_{d}$, where the symmetric group $\Sigma_{d}$ acts on the $d$-fold product $X^{d}$ in the natural way. An element of $\operatorname{SP}^{d}(X)$ may be identified with a formal linear combination $\alpha=\sum_{i=1}^{k} d_{i} x_{i}$, where $x_{1}, \ldots, x_{k}$ are distinct points of $X$ and $d_{1}, \ldots, d_{k}$ are positive integers such that $\sum_{i=1}^{k} d_{i}=d$. We shall refer to $\alpha$ as a "configuration" of points, the point $x_{i}$ having multiplicity $d_{i}$. We shall be concerned with a subspace $\operatorname{SP}_{n}^{d}(X)$ of $\operatorname{SP}^{d}(X)$, defined as follows. If $n \geq 2$,

$$
\mathrm{SP}_{n}^{d}(X)=\left\{\sum_{i=1}^{k} d_{i} x_{i} \in \mathrm{SP}^{d}(X): d_{i}<n \text { for all } i\right\} .
$$

Thus, $\mathrm{SP}_{n}^{d}(X)$ is obtained by imposing a condition of "bounded multiplicity", namely that all points $x_{i}$ (of any configuration) have multiplicity less than $n$. There is a filtration

$$
C_{d}(X)=\operatorname{SP}_{2}^{d}(X) \subset \cdots \subset \operatorname{SP}_{n}^{d}(X) \subset \cdots \subset \operatorname{SP}_{d+1}^{d}(X)=\cdots=\operatorname{SP}^{d}(X),
$$

where $C_{d}(X)$ denotes the space of "configurations of $d$ distinct points" in $X$. Note that $\mathrm{SP}_{n}^{d}(\mathbb{C})$ can be identified with the space of complex polynomials of degree $d$ which are monic, and all of whose roots have multiplicity less than $n$. (The polynomial $\prod_{i=1}^{k}\left(z-x_{i}\right)^{d_{i}}$ corresponds to $\sum_{i=1}^{k} d_{i} x_{i}$.)

If we choose the point $[1: \cdots: 1]$ as the base point of $\mathbb{C P}^{m-1}$, the space $\operatorname{Hol}_{d}^{*}\left(S^{2}, \mathbb{C P}^{m-1}\right)$ is identified with the space consisting of all $m$-tuples $\left(p_{1}(z), \ldots, p_{m}(z)\right) \in \mathbb{C}[z]^{m}$ of monic polynomials of degree $d$ such that the polynomials $p_{1}(z), \ldots, p_{m}(z)$ have no common roots. It is also identified with the space of $m$-tuples of positive disjoint divisors,

$$
\operatorname{Hol}_{d}^{*}\left(S^{2}, \mathbb{C P}^{m-1}\right)=\left\{\left(\xi_{1}, \ldots, \xi_{m}\right) \in \operatorname{SP}^{d}(\mathbb{C})^{m}: \xi_{1} \cap \cdots \cap \xi_{m}=\emptyset\right\} .
$$

In this situation, let $\operatorname{Hol}_{d}^{n}\left(S^{2}, \mathbb{C P}^{m-1}\right)$ denote the subspace defined by

$$
\begin{aligned}
\operatorname{Hol}_{d}^{n}\left(S^{2}, \mathbb{C P}^{m-1}\right) & =\operatorname{Hol}_{d}^{*}\left(S^{2}, \mathbb{C P}^{m-1}\right) \cap \mathrm{SP}_{n}^{d}(\mathbb{C})^{m} \\
& =\left\{\left(\xi_{1}, \ldots, \xi_{m}\right) \in \operatorname{SP}_{n}^{d}(\mathbb{C})^{m}: \xi_{1} \cap \cdots \cap \xi_{m}=\emptyset\right\} .
\end{aligned}
$$

Now we recall the following result. 
Theorem 1.2 ([13]). If $n \geq 2$, there is a map $\operatorname{Hol}_{d}^{n}\left(S^{2}, \mathbb{C P}^{m-1}\right) \rightarrow$ $\Omega_{0}^{2} W_{m}\left(\mathbb{C P}^{n-1}\right)$ which is a homology equivalence up to $[d / 2]$ when $n=2$ and a homotopy equivalence up to dimension $D_{1}(d ; m, n)=d-2 m+2$ when $n \geq 3$, where $[x]$ denotes the integer part of a number $x$ and $W_{m}(X) \subset X^{m}$ denotes the $m$-th fat wedge.

In this paper, we would like to generalize the above result for another wider complex projective varieties. For this purpose, let $m, n \geq 2$ be integers and let $X_{m} \subset \mathbb{C P}^{m-1}$ denote the quasi-projective variety defined by

$$
X_{m}=\mathbb{C P}^{m-1}-\bigcup_{1 \leq i<j \leq m} H_{i, j},
$$

where $H_{i, j}=\left\{\left[z_{1}: \cdots: z_{m}\right] \in \mathbb{C P}^{m-1}: z_{i}=z_{j}=0\right\}$.

It is known [5] that $X_{m}$ is simply connected and that $\pi_{2}\left(X_{m}\right)=\mathbb{Z}$. If we choose the point $[1: \cdots: 1]$ as the base point of $X_{m}, \operatorname{Hol}_{d}^{*}\left(S^{2}, X_{m}\right)$ is identified with the space consisting of all $m$-tuples $\left(p_{1}(z), \ldots, p_{m}(z)\right) \in \mathbb{C}[z]^{m}$ of mutually coprime monic polynomials of degree $d$. We can also identify

$$
\operatorname{Hol}_{d}^{*}\left(S^{2}, X_{m}\right)=\left\{\left(\xi_{1}, \ldots, \xi_{m}\right) \in \operatorname{SP}^{d}(\mathbb{C})^{m}: \xi_{i} \cap \xi_{j}=\emptyset \text { if } i \neq j\right\} .
$$

We denote by $\operatorname{Hol}_{d}^{n}\left(S^{2}, X_{m}\right)$ the subspace of $\operatorname{Hol}_{d}^{*}\left(S^{2}, X_{m}\right)$ defined by

$$
\begin{aligned}
\operatorname{Hol}_{d}^{n}\left(S^{2}, X_{m}\right) & =\operatorname{Hol}_{d}^{*}\left(S^{2}, X_{m}\right) \cap \operatorname{SP}_{n}^{d}(\mathbb{C})^{m} \\
& =\left\{\left(\xi_{1}, \ldots, \xi_{m}\right) \in \operatorname{SP}_{n}^{d}(\mathbb{C})^{m}: \xi_{i} \cap \xi_{j}=\emptyset \text { if } i \neq j\right\} .
\end{aligned}
$$

There is a filtration

$$
\begin{aligned}
& \emptyset=\operatorname{Hol}_{d}^{1}\left(S^{2}, X_{m}\right) \subset \operatorname{Hol}_{d}^{2}\left(S^{2}, X_{m}\right) \subset \operatorname{Hol}_{d}^{3}\left(S^{2}, X_{m}\right) \subset \cdots \\
& \subset \operatorname{Hol}_{d}^{d+1}\left(S^{2}, X_{m}\right)=\operatorname{Hol}_{d}^{d+2}\left(S^{2}, X_{m}\right)=\cdots=\operatorname{Hol}_{d}^{*}\left(S^{2}, X_{m}\right) .
\end{aligned}
$$

Let $\vee^{m} X$ denote the $m$-times wedge of $X, \vee^{m} X=X \vee X \vee \cdots \vee X$ ( $m$-times). Then our main result is stated as follows.

Theorem 1.3. If $m, n \geq 2$, there is a map $\operatorname{Hol}_{d}^{n}\left(S^{2}, X_{m}\right) \rightarrow \Omega_{0}^{2} \vee^{m}$ $\mathbb{C P}^{n-1}$ which is a homotopy equivalence up to dimension $D(d ; m, n)$ when $n \geq 3$ and a homology equivalence up to dimension $[d / 2]$ when $n=2$. Here the number $D(d ; m, n)$ is given by

$$
D(d ; m, n)= \begin{cases}d+n-4 & \text { if } d \geq n \\ d & \text { if } d<n .\end{cases}
$$


Remark. (1) Since $\lim _{d \rightarrow \infty} D(d ; m, n)=\infty$, the space $\operatorname{Hol}_{d}^{n}\left(S^{2}, X_{m}\right)$ may be regarded as a finite dimensional model for the infinite dimensional space $\Omega_{0}^{2}\left(\vee^{m} \mathbb{C P}^{n-1}\right)$.

(2) It follows from [5] that there is a fibration sequence $T^{n} \rightarrow X_{m} \rightarrow$ $\vee^{m} \mathbb{C P}^{\infty}$. Hence, using an easy diagram chasing as in [[13]; page 251] we obtain a homotopy equivalence $\lim _{d, n \rightarrow \infty} \operatorname{Hol}_{d}^{n}\left(S^{2}, X_{m}\right) \simeq \Omega_{0}^{2} X_{m}$. So we obtain the stabilized version of the main theorem of [5] if $n \rightarrow \infty$.

Next we shall indicate the following generalization of Theorem 1.3 to the case of certain quasi-projective varieties $X_{I}$.

Let $I$ be a fixed collections of subsets of $\{1,2, \ldots, m\}$ such that $\operatorname{card}(\Lambda) \geq 2$ for any $\Lambda \in I$. We denote by $X_{I} \subset \mathbb{C P}^{m-1}$ the quasi-projective variety defined by

$$
X_{I}=\mathbb{C P}^{m-1}-\bigcup_{\Lambda \in I} H_{\Lambda},
$$

where $H_{\Lambda}=\left\{\left[z_{1}: \cdots: z_{m}\right] \in \mathbb{C P}^{m-1}: z_{i}=0\right.$ for all $\left.i \in \Lambda\right\}$.

It is known [5] that $X_{I}$ is simply connected, $\pi_{2}\left(X_{I}\right)=\mathbb{Z}$, and that there is a fibration sequence $T^{m-1}=\left(S^{1}\right)^{m-1} \rightarrow X_{I} \stackrel{q_{I}}{\rightarrow} \vee^{I} \mathbb{C P}^{\infty}$, where $\vee^{I} X$ denotes the wedge of type $I$ given by $\vee^{I} X=\left\{\left(x_{1}, \ldots, x_{m}\right) \in X^{m}\right.$ : for each $\Lambda \in I, x_{i}=$ $*$ for some $i \in \Lambda$ \}.

Example. (1) If $I=\{\{1,2, \ldots, m\}\}, X_{I}=\mathbb{C P}^{m-1}$ and $\vee^{I} X=W_{m}(X)$.

(2) If $I=\{\{i, j\}: 1 \leq i<j \leq m\}, X_{I}=X_{m}$ and $\vee^{I} X=\vee^{m} X$.

If we choose the point $[1: \cdots: 1] \in X_{I}$ as a base point of $X_{I}$, we can identify $\operatorname{Hol}_{d}^{*}\left(S^{2}, X_{I}\right)=\left\{\left(\xi_{1}, \ldots, \xi_{m}\right) \in \operatorname{SP}^{d}(\mathbb{C})^{m}: \cap_{i \in \Lambda} \xi_{i}=\emptyset\right.$ for any $\left.\Lambda \in I\right\}$. Then we denote by $\operatorname{Hol}_{d}^{n}\left(S^{2}, X_{I}\right)$ the subspace of $\operatorname{Hol}_{d}^{*}\left(S^{2}, X_{I}\right)$ defined by

$$
\begin{aligned}
\operatorname{Hol}_{d}^{n}\left(S^{2}, X_{I}\right) & =\operatorname{Hol}_{d}^{*}\left(S^{2}, X_{I}\right) \cap \operatorname{SP}_{n}^{d}(\mathbb{C})^{m} \\
& =\left\{\left(\xi_{1}, \ldots, \xi_{m}\right) \in \operatorname{SP}_{n}^{d}(\mathbb{C})^{m}: \cap_{i \in \Lambda} \xi_{i}=\emptyset \text { for any } \Lambda \in I\right\} .
\end{aligned}
$$

There is a filtration

$$
\begin{aligned}
& \emptyset=\operatorname{Hol}_{d}^{1}\left(S^{2}, X_{I}\right) \subset \operatorname{Hol}_{d}^{2}\left(S^{2}, X_{I}\right) \subset \operatorname{Hol}_{d}^{3}\left(S^{2}, X_{I}\right) \subset \cdots \\
& \subset \operatorname{Hol}_{d}^{d+1}\left(S^{2}, X_{I}\right)=\operatorname{Hol}_{d}^{d+2}\left(S^{2}, X_{I}\right)=\cdots=\operatorname{Hol}_{d}^{*}\left(S^{2}, X_{I}\right) .
\end{aligned}
$$

Theorem 1.4. Let $m, n \geq 2$ be integers and $I$ be a fixed collections of subsets of $\{1,2, \ldots, m\}$ such that $\operatorname{card}(\Lambda) \geq 2$ for each $\Lambda \in I$. Then there is a map $\operatorname{Hol}_{d}^{n}\left(S^{2}, X_{I}\right) \rightarrow \Omega_{0}^{2} \vee^{I} \mathbb{C P}^{n-1}$ which is a homotopy equivalence up to dimension $D(d ; m, n)$ when $n \geq 3$ and a homology equivalence up to dimension $[d / 2]$ when $n=2$. Here the number $D(d ; m, n)$ is same as in (1.3.1). 
Since $X_{I}=\mathbb{C P}^{m-1}$ if $I=\{\{1,2, \ldots, m\}\}$ and $D_{1}(d ; m, n)<D(d ; m, n)$ when $n>2$, the above theorem indicates that the main result of [13] can be improved. Moreover, although the proof given in [13] only works for the case $X=\mathbb{C P}^{m-1}$, the proof given in this paper works for all $X_{I}$ (and $\mathbb{C P}^{m-1}$, too). So this permit us not only to sharpen the stability dimension, but also, more importantly, to obtain the stability result for wider quasi-projective varieties.

Our results may confirm this Morse theory principle for another varieties (e.g. various toric varieties cf. [8]). The explanation for these type theorems is thought to be Morse theoretic in nature, i.e. the fact that the holomorphic maps form the set of absolute minima of the energy functional on (a fixed component of) the space of smooth maps. However, the existing proofs of these results are topological in nature (cf. [3], [4]).

This paper is organized as follows. In section 2, we shall recall the stabilized theorem using scanning method originally invented by G. Segal [11]. In section 3 , we shall give the proof of Theorem 1.3. To prove this we shall show Theorem 3.3, which is the key of this paper. In section 4 , we shall indicate how the proof of Theorem 3.3 can be generalized and give the proof of Theorem 1.4. Finally, in section 5 , we shall study the stability of $s^{d}: \mathrm{SP}_{n}^{d}\left(\mathbb{C}_{k}\right) \rightarrow \mathrm{SP}_{n}^{d+1}\left(\mathbb{C}_{k}\right)$ using the geometric resolution ([12]) and we shall give the proof of Theorem 5.1, which will be used in the proof of Theorem 3.3.

\section{$\S 2 . \quad$ Stabilized Spaces}

In this section, we recall the scanning map and its basic properties. Because this was now well explained in several papers (cf. [5], [6], [7], [8], [10], [11]), we only sketch the rough idea.

Let $I$ be a collection of subsets of $\{1,2, \ldots, m\}$ such that card $(\Lambda) \geq 2$ for any $\Lambda \in I$. For a connected space $X$, let $E_{d}^{I, n}(X)$ denote the space defined by $E_{d}^{I, n}(X)=\left\{\left(\xi_{1}, \ldots, \xi_{m}\right) \in \operatorname{SP}_{n}^{d}(X)^{m}: \cap_{i \in \Lambda} \xi_{i}=\emptyset\right.$ for any $\left.\Lambda \in I\right\}$.

Remark that $E_{d}^{I, n}(\mathbb{C})=\operatorname{Hol}_{d}^{n}\left(S^{2}, X_{I}\right)$ if $X=\mathbb{C}$. If $A \subset X$ is a closed subspace, we define the relative configuration space $E_{d}^{I, n}(X, A)=E_{d}^{I, n}(X) / \sim$, where $\left(\xi_{1}, \ldots, \xi_{m}\right) \sim\left(\eta_{1}, \ldots, \eta_{m}\right)$ if and only if $\xi_{j} \cap(X-A)=\eta_{j} \cap(X-A)$ for each $1 \leq j \leq m$. Thus, for each $E_{d}^{I, n}(X, A)$, points in $A$ are ignored. When $A \neq \emptyset$, there is a natural inclusion $E_{d}^{I, n}(X, A) \rightarrow E_{d+1}^{I, n}(X, A)$ given by adding points in $A$. We define $E^{I, n}(X, A)=\bigcup_{d \geq 1} E_{d}^{I, n}(X, A)$.

Let $s_{d}: E_{d}^{I, n}(\mathbb{C}) \rightarrow E_{d+1}^{I, n}(\mathbb{C})$ denote the stabilization map given by adding a point from the edge in a usual way (see [5], [6], [8], [11]), and let $\lim _{d \rightarrow \infty} E_{d}^{I, n}(\mathbb{C})$ the colimit space induced from stabilization maps $s_{d}$. 
Let $\lim _{d \rightarrow \infty} s_{n}^{d}: \lim _{d \rightarrow \infty} E_{d}^{I, n}(\mathbb{C}) \rightarrow \Omega_{0}^{2} E^{I, n}\left(S^{2}, \infty\right)$ be the scanning map defined similarly as [[6]; page 99]. If we identify $E_{d}^{I, n}(\mathbb{C})=\operatorname{Hol}_{d}^{n}\left(S^{2}, X_{I}\right)$, then we have the scanning map $S: \lim _{d \rightarrow \infty} \operatorname{Hol}_{d}^{n}\left(S^{2}, X_{I}\right) \rightarrow \Omega_{0}^{2} E^{I, n}\left(S^{2}, \infty\right)$.

Theorem 2.1. $\quad S: \lim _{d \rightarrow \infty} \operatorname{Hol}_{d}^{n}\left(S^{2}, X_{I}\right) \stackrel{\simeq}{\rightrightarrows} \Omega_{0}^{2} E^{I, n}\left(S^{2}, \infty\right)$ is a homotopy equivalence when $n \geq 3$ and a homology equivalence when $n=2$.

Proof. This is similar to the proof in section 3 of [11] (cf. [7]).

Lemma 2.1. There is a homotopy equivalence $E^{I, n}\left(S^{2}, \infty\right) \simeq \vee^{I} \mathbb{C P}^{n-1}$.

Proof. If $I=\{\{i, j\}: 1 \leq i<j \leq n\}$, the proof is completely same as that of [[5]; Proposition 3.2]. For a general case, the similar method may be used and we omit the detail.

Corollary 2.1. $\quad S: \lim _{d \rightarrow \infty} \operatorname{Hol}_{d}^{n}\left(S^{2}, X_{I}\right) \stackrel{\cong}{\rightrightarrows} \Omega_{0}^{2} \vee^{I} \mathbb{C P}^{n-1}$ is a homotopy equivalence when $n \geq 3$ and $a \stackrel{d \rightarrow \infty}{h o m o l o g y}$ equivalence when $n=2$.

\section{$\S 3 . \quad$ Unstability Result}

Theorem 3.1. Let $k \geq 1$ be an integer and let $\mathbb{C}_{k}=\mathbb{C}-\{1,2, \ldots, k\}$. If $d \geq n \geq 3$, the stabilization map $s^{d}: \mathrm{SP}_{n}^{d}\left(\mathbb{C}_{k}\right) \rightarrow \mathrm{SP}_{n}^{d+1}\left(\mathbb{C}_{k}\right)$ is a homotopy equivalence up to dimension $N(d, n)=d+n-4=D(d ; m, n)$.

The proof of Theorem 3.1 is postponed to the last section and we prove the following result.

Theorem 3.2. If $m, n \geq 2, s_{d}: \operatorname{Hol}_{d}^{n}\left(S^{2}, X_{m}\right) \rightarrow \operatorname{Hol}_{d+1}^{n}\left(S^{2}, X_{m}\right)$ is a homotopy equivalence up to dimension $D(d ; m, n)$ when $n \geq 3$ and a homology equivalence up to dimension $[d / 2]$ when $n=2$. Here the number $D(d ; m, n)$ is same as in (1.3.1).

Before proving Theorem 3.2, we complete the proof of Theorem 1.3.

Proof of Theorem 1.3. The assertion easily follows from Corollary 2.1 and Theorem 3.2.

Definition. Let $V \subset \mathbb{C}$ be an open set and $d_{1}, \ldots, d_{m} \geq 1$ be integers. Let $E_{d_{1}, \ldots, d_{m}}^{n}(V)$ be the subspace of $\operatorname{SP}_{n}^{d_{1}}(V) \times \cdots \times \operatorname{SP}_{n}^{d_{m}}(V)$ defined by

$$
E_{d_{1}, \ldots, d_{m}}^{n}(V)=\left\{\left(\xi_{1}, \ldots, \xi_{m}\right): \xi_{i} \in \operatorname{SP}_{n}^{d_{i}}(V) \text { for each } i, \xi_{i} \cap \xi_{j}=\emptyset \text { if } i \neq j\right\} .
$$


If $d_{1}=d_{2}=\cdots=d_{m}=d$, we write $E_{d}^{n}(V)=E_{d_{1}, \ldots, d_{m}}^{n}(V)$. If $V=\mathbb{C}$, we also write $E_{d_{1}, \ldots, d_{m}}^{n}=E_{d_{1}, \ldots, d_{m}}^{n}(\mathbb{C})$.

Let $z_{1}, \ldots, z_{m} \in\{w \in \mathbb{C}: 1<|w|<2\}$ be any fixed points such that $z_{i} \neq z_{j}$ if $i \neq j$. For each $1 \leq k \leq m$, define the map

$$
j_{k}: E_{d_{1}, \ldots, d_{k}, \ldots, d_{m}}^{n}(\{|w|<1\}) \rightarrow E_{d_{1}, \ldots, d_{k-1}, d_{k}+1, d_{k+1} \ldots, d_{m}}^{n}(\{|w|<2\})
$$

by $\left(\xi_{1}, \ldots, \xi_{m}\right) \mapsto\left(\xi_{1}, \ldots, \xi_{k-1}, \xi_{k}+z_{k}, \xi_{k+1}, \ldots, \xi_{m}\right)$. Up to homotopy, $j_{k}$ defines the map $j_{k}: E_{d_{1}, \ldots, d_{k}, \ldots, d_{m}}^{n} \rightarrow E_{d_{1}, \ldots, d_{k-1}, d_{k}+1, d_{k+1}, \ldots, d_{m}}^{n}$.

Theorem 3.3. Let $m, n \geq 2,1 \leq k \leq m$, and $d_{1}, \ldots, d_{m} \geq 2$ are integers. Then $j_{k}: E_{d_{1}, \ldots, d_{k}, \ldots, d_{m}}^{n} \rightarrow E_{d_{1}, \ldots, d_{k-1}, d_{k}+1, d_{k+1}, \ldots, d_{m}}^{n}$ is a homotopy equivalence up to dimension $D\left(d_{k} ; m, n\right)$ when $n \geq 3$ and a homology equivalence up to dimension $\left[d_{k} / 2\right]$ when $n=2$.

Before giving the proof of Theorem 3.3, we complete the proof of Theorem 3.2 .

Proof of Theorem 3.2. If we may identify $\operatorname{Hol}_{d}^{n}\left(S^{2}, X_{m}\right)=E_{d}^{n}(\mathbb{C})$, we have $s_{d}=j_{1} \circ j_{2} \circ \cdots \circ j_{m}$ (up to homotopy). Hence the assertion easily follows from Theorem 3.3.

Definition. We say that a map $f: X \rightarrow Y$ is acyclic up to dimension $N$ if for any local coefficient system $L$ on $Y, f_{*}: H_{i}\left(X, f^{*} L\right) \rightarrow H_{i}(Y, L)$ is bijective when $i<N$ and is surjective when $i=N$, where $f^{*} L$ is the induced local system on $X$.

Lemma $3.1([9])$. Let $f: X \rightarrow Y$ be a continuous map between connected $C W$ complexes such that $\pi_{1}(X)$ and $\pi_{1}(Y)$ are abelian groups. If the map $f$ is acyclic up to dimension $N$, it is a homotopy equivalence up to dimen$\operatorname{sion} N$.

Lemma 3.2. If $d_{1}, \ldots, d_{m} \geq 2$ and $n \geq 3$ are integers, $\pi_{1}\left(E_{d_{1}, \ldots, d_{m}}^{n}\right)$ is an abelian group.

Proof. Geometrically $\pi_{1}\left(E_{d_{1}, \ldots, d_{m}}^{n}\right)$ may described as the group of $m$ tuples ( $d_{1}$-strings, $d_{2}$-strings, $\cdots, d_{m}$-strings) of braids such that $i$-th braids are allowed to pass until $(n-1)$-crossings. Hence, the assertion may be proved in a similar way as in [[5]; appendix].

Now we can give the proof of Theorem 3.3. 
Proof of Theorem 3.3. It suffices to give the proof when $k=1$. Let $\pi: E_{d_{1}, \ldots, d_{m}}^{n} \rightarrow E_{d_{2}, \ldots, d_{m}}^{n}$ be the projection map given by $\pi\left(\xi_{1}, \ldots, \xi_{m}\right)=$ $\left(\xi_{2}, \ldots, \xi_{m}\right)$. If $n \geq 3$ and $d_{1}<n$, Theorem 3.3 follows from [[5]; Theorem 1.8] and Lemmas 3.1, 3.2. If $n=2$, we have a homotopy commutative diagram

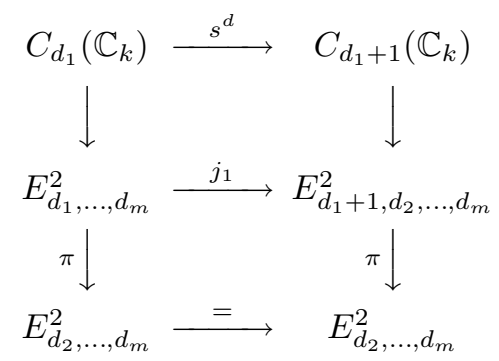

where we take $k=\sum_{i=2}^{m} d_{i}$ and $\mathbb{C}_{k}=\mathbb{C}-\{1,2, \ldots, k\}$, vertical sequences are fibrations and $s^{d}$ is a stabilization map. Since $s^{d}$ is a homology equivalence up to dimension $\left[d_{1} / 2\right]$ ([[11]; appendix] $\left.]\right), j_{1}$ is also a homology equivalence up to dimension $\left[d_{1} / 2\right]$. Hence the assertion holds when $n=2$.

So without loss of generalities, we may assume that $d_{1} \geq n \geq 3$. Then it follows from Lemmas $3.1,3.2$ that it suffices to show that $j_{1}$ is acyclic up to dimension $N\left(d_{1}, n\right)=d_{1}+n-4$. Let $L$ be a local coefficient system on $E_{d_{1}+1, d_{2}, \ldots, d_{m}}^{n}$. We shall use the letter $L$ to denote its restriction to any (closed or open) subspace. In this case, we also consider the commutative diagram

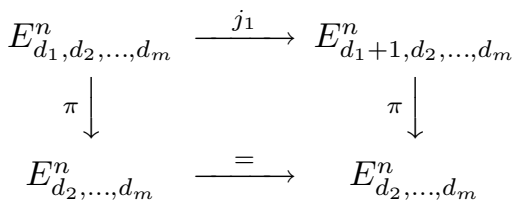

If vertical maps were fibrations, we could prove the assertion in a similar way as above. However, they are fibrations only over certain subspaces as we shall now explain.

Since $E_{d_{1}, d_{2}, \ldots, d_{m}}^{n} \subset \mathrm{SP}^{d_{1}}(\mathbb{C}) \times \cdots \times \mathrm{SP}^{d_{m}}(\mathbb{C}) \cong \mathbb{C}^{d_{1}} \times \cdots \times \mathbb{C}^{d_{m}}=\mathbb{C}^{d}$ is an open set, $E_{d_{1}, d_{2}, \ldots, d_{m}}^{n}$ is an open complex manifold of dimension $d=\sum_{i=1}^{m} d_{i}$ and the Poincaré duality isomorphism $H_{k}\left(E_{d_{1}, d_{2}, \ldots, d_{m}}^{n} ; L\right) \cong H_{c}^{2 d-k}\left(E_{d_{1}, d_{2}, \ldots, d_{m}}^{n}\right.$; $H(L))$ holds, where $H(L)$ denotes the orientation bundle and $H_{c}^{i}$ denotes the cohomology with compact supports $([2])$.

From now on, we may identify $E_{d_{1}, d_{2}, \ldots, d_{m}}^{n}$ with the space consisting of all $m$-tuples $\left(p_{1}(z), \ldots, p_{m}(z)\right) \in \mathbb{C}[z]^{m}$ of monic polynomials satisfying the following two conditions: 
(a) $p_{i}(z)$ is a monic polynomial of degree $d_{i}$ and has no roots of multiplicity $\geq n$ for each $1 \leq i \leq m$.

(b) $p_{i}(z)$ and $p_{k}(z)$ are coprime whenever $i \neq k$.

Let $E_{d_{1}, d_{2}, \ldots, d_{m}}^{k_{1}, k_{2}, \ldots, k_{m}}$ be the subspace of $E_{d_{1}, d_{2}, \ldots, d_{m}}^{n}$ consisting of all $m$-tuples of polynomials $\left(p_{1}(z), \ldots, p_{m}(z)\right) \in E_{d_{1}, d_{2}, \ldots, d_{m}}^{n}$ which satisfies the following condition:

(c) $p_{j}(z)$ has at least $k_{j}$ distinct roots for each $1 \leq j \leq m$.

Let $X_{d_{1}, d_{2}, \ldots, d_{m}}^{1, k_{2}, \ldots, k_{m}}=E_{d_{1}, d_{2}, \ldots, d_{m}}^{1, k_{2}, \ldots, k_{m}}-\bigcup_{i=2}^{m} E_{d_{1}, d_{2}, \ldots, d_{i}, \ldots, d_{m}}^{1, k_{2}, \ldots, k_{i}+1, \ldots, k_{m}}$, i.e. the subset consisting of all $m$-tuples $\left(p_{1}(z), \ldots, p_{m}(z)\right) \in E_{d_{1}, \ldots, d_{m}}^{n}$ such that each $p_{i}(z)(2 \leq i \leq m)$ has exactly $k_{i}$ distinct roots.

Let $Y_{d_{2}, \ldots, d_{m}}^{k_{2}, \ldots, k_{m}}=\pi\left(X_{d_{1}, d_{2}, \ldots, d_{m}}^{1, k_{2}, \ldots, k_{m}}\right)=E_{d_{2}, \ldots, d_{m}}^{k_{2}, \ldots, k_{m}}-\bigcup_{i=2}^{m} E_{d_{2}, \ldots, d_{i}, \ldots, d_{m}}^{k_{2}, \ldots, k_{i}+1, \ldots, k_{m}}$. The map $\pi$ restricts to a map $\pi: X_{d_{1}, d_{2}, \ldots, d_{m}}^{1, k_{2}, \ldots, k_{m}} \rightarrow Y_{d_{2}, \ldots, d_{m}}^{k_{2}, \ldots, k_{m}}$ and we obtain the homotopy commutative diagram

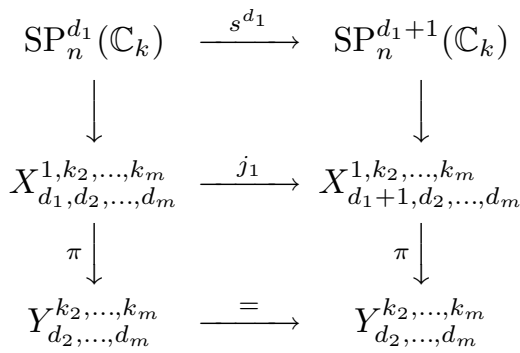

where we take $k=\sum_{i=2}^{m} k_{i}, \mathbb{C}_{k}=\mathbb{C}-\{1,2, \ldots, k\}$, and vertical sequences are fibrations.

Since $s^{d_{1}}$ is a homotopy equivalence up to dimension $N\left(d_{1}, n\right)$ by Theorem 3.1, $j_{1}: X_{d_{1}, d_{2}, \ldots, d_{m}}^{1, k_{2}, \ldots, k_{m}} \rightarrow X_{d_{1}+1, d_{2}, \ldots, d_{m}}^{1, k_{2}, \ldots, k_{m}}$ is also a homotopy equivalence up to dimension $N\left(d_{1}, n\right)$. Now we remark the following:

$\left(\dagger_{k}\right)$ If $1 \leq k_{i} \leq d_{i}(i=2, \ldots, m)$, then $j_{1}: E_{d_{1}, d_{2}, \ldots, d_{m}}^{1, k_{2}, \ldots, k_{m}} \rightarrow E_{d_{1}+1, d_{2}, \ldots, d_{m}}^{1, k_{2}, \ldots, k_{m}}$ is acyclic up to dimension $N\left(d_{1}, n\right)$.

We postpone the poof of $\left(\dagger_{k}\right)$ and complete the proof of 3.3. If $\left(\dagger_{k}\right)$ is true, then $j_{1}: E_{d_{1}, d_{2}, \ldots, d_{m}}^{1,1, \ldots, 1} \rightarrow E_{d_{1}+1, d_{2}, \ldots, d_{m}}^{1,1, \ldots, 1}$ is acyclic up to dimension $N\left(d_{1}, n\right)$. Since $E_{d_{1}, d_{2}, \ldots, d_{m}}^{1,1, \ldots, 1}=E_{d_{1}, d_{2}, \ldots, d_{m}}^{n}, j_{1}: E_{d_{1}, d_{2}, \ldots, d_{m}}^{n} \rightarrow E_{d_{1}+1, d_{2}, \ldots, d_{m}}^{n}$ is acyclic up to dimension $N\left(d_{1}, n\right)$. This completes the proof of Theorem 3.3. 
Proof of $\left(\dagger_{k}\right)$ : The proof of $\left(\dagger_{k}\right)$ is by downwards induction on $k=\sum_{i=2}^{m} k_{i}$. The induction begins with $k=\sum_{i=2}^{m} d_{i}$. Since $E_{d_{1}, d_{2}, \ldots, d_{m}}^{1, d_{2}, \ldots, d_{m}}=X_{d_{1}, d_{2}, \ldots, d_{m}}^{1, d_{2}, \ldots, d_{m}}$, the assertion is clearly true. As a next step, we assume that $\left(\dagger_{l}\right)$ holds for any $l>k$ and we shall prove that $\left(\dagger_{k}\right)$ is true. We remark that for any $2 \leq j \leq m$ the following statement holds:

$\left(\ddagger_{j}\right) j_{1}: \bigcup_{i=1}^{j} E_{d_{1}, d_{2}, \ldots, d_{m}}^{1, l_{2}(i), \ldots, l_{m}(i)} \rightarrow \bigcup_{i=1}^{j} E_{d_{1}+1, d_{2}, \ldots, d_{m}}^{1, l_{2}(i), \ldots, l_{m}(i)}$ is a homology equivalence up to dimension $N\left(d_{1}, n\right)$ whenever $\sum_{t=2}^{m} l_{t}(i)>k$ for any $1 \leq j \leq m$.

Proof of $\left(\ddagger_{j}\right)$ : We can prove $\left(\ddagger_{j}\right)$ easily by induction on $j$. In fact, if $j=1$, $\left(\ddagger_{j}\right)$ holds, because $\left(\dagger_{l}\right)$ is true when $l>k$. Now assume $\left(\ddagger_{j-1}\right)$ is true and we shall show that $\left(\ddagger_{j}\right)$ is true. Since $E_{d_{1}, d_{2}, \ldots, d_{m}}^{1, k_{2}, \ldots, k_{m}} \cap E_{d_{1}, d_{2}, \ldots, d_{m}}^{1, k_{2}^{\prime}, \ldots, k_{m}^{\prime}}=$ $E_{d_{1}, d_{2}, \ldots, d_{m}}^{1, \max \left(k_{2}, k_{2}^{\prime}\right), \ldots, \max \left(k_{m}, k_{m}^{\prime}\right)}$, using the Mayer-Vietoris exact sequence and 5-lemma, we can prove the assertion $\left(\ddagger_{j}\right)$ easily.

It follows from $\left(\ddagger_{\ddagger}\right)$ that $j_{1}: \bigcup_{i=2}^{m} E_{d_{1}, d_{2}, \ldots, d_{i}, \ldots, d_{m}}^{1, k_{2}, \ldots, k_{i}+1, \ldots, k_{m}} \rightarrow \bigcup_{i=2}^{m} E_{d_{1}+1, d_{2}, \ldots, d_{i}, \ldots, d_{m}}^{1, k_{2}, \ldots, k_{i}+1, \ldots, k_{m}}$ is a homology equivalence up to dimension $N\left(d_{1}, n\right)$.

Since $E_{d_{1}, d_{2}, \ldots, d_{i}, \ldots, d_{m}}^{1, k_{2}, \ldots, k_{i}+1, \ldots, k_{m}} \subset E_{d_{1}, d_{2}, \ldots, d_{i}, \ldots, d_{m}}^{n}$ is an open subspace, the space $\bigcup_{i=2}^{m} E_{d_{1}, d_{2}, \ldots, d_{i}, \ldots, d_{m}}^{1, k_{2}, \ldots, k_{i}+1, \ldots, k_{m}}$ is an open complex manifold of dimension $d$. Hence it follows from Poincaré duality that there is a commutative diagram

$$
\begin{array}{r}
H_{j}\left(\bigcup_{i=2}^{m} E_{d_{1}, d_{2}, \ldots, d_{i}, \ldots, d_{m}}^{1, k_{2}, \ldots, k_{i}+1, \ldots, k_{m}} ; L\right) \stackrel{j_{1 *}}{\longrightarrow} H_{j}\left(\bigcup_{i=2}^{m} E_{d_{1}+1, d_{2}, \ldots, d_{i}, \ldots, d_{m}}^{1, k_{2}, \ldots, k_{i}+1, \ldots, k_{m}} ; L\right) \\
\cong \downarrow \\
H_{c}^{2 d-j}\left(\bigcup_{i=2}^{m} E_{d_{1}, d_{2}, \ldots, d_{i}, \ldots, d_{m}}^{1, k_{2}, \ldots, k_{i}+1, \ldots, k_{m}}\right) \stackrel{j_{1}{ }^{*}}{\longrightarrow} H_{c}^{2 d+2-j}\left(\bigcup_{i=2}^{m} E_{d_{1}+1, d_{2}, \ldots, d_{i}, \ldots, d_{m}}^{1, k_{2}, \ldots, k_{i}+d_{2}, \ldots, k_{m}}\right)
\end{array}
$$

where $H_{c}^{k}(Y)=H_{c}^{k}(Y ; H(L))$. Hence, the induced homomorphism

$$
j_{1}{ }^{*}: H_{c}^{j}\left(\bigcup_{i=2}^{m} E_{d_{1}, d_{2}, \ldots, d_{i}, \ldots, d_{m}}^{1, k_{2}, \ldots, k_{i}+1, \ldots, k_{m}}\right) \rightarrow H_{c}^{j+2}\left(\bigcup_{i=2}^{m} E_{d_{1}+1, d_{2}, \ldots, d_{i}, \ldots, d_{m}}^{1, k_{2}, \ldots, k_{i}+1, \ldots, k_{m}}\right)
$$

is bijective when $j>2 d-N\left(d_{1}, n\right)$ and surjective when $j=2 d-N\left(d_{1}, n\right)$.

Similarly, because $j_{1}: X_{d_{1}, d_{2}, \ldots, d_{m}}^{1, k_{2}, \ldots, k_{m}} \rightarrow X_{d_{1}+1, d_{2}, \ldots, d_{m}}^{1, k_{2}, \ldots, k_{m}}$ is also a homotopy equivalence up to dimension $N\left(d_{1}, n\right)$, the induced homomorphism

$$
j_{1}^{*}: H_{c}^{j}\left(X_{d_{1}, d_{2}, \ldots, d_{m}}^{1, k_{2}, \ldots, k_{m}}\right) \rightarrow H_{c}^{j+2}\left(X_{d_{1}+1, d_{2}, \ldots, d_{m}}^{1, k_{2}, \ldots, k_{m}}\right)
$$

is bijective when $j>2 d-N\left(d_{1}, n\right)$ and surjective when $j=2 d-N\left(d_{1}, n\right)$. 
Consider the cohomology exact sequence with compact supports of the pair $\left(E_{d_{1}, d_{2}, \ldots, d_{m}}^{1, k_{2}, \ldots, k_{m}}, \bigcup_{i=2}^{m} E_{d_{1}, d_{2}, \ldots, d_{i}, \ldots, d_{m}}^{1, k_{2}, \ldots, k_{i}+1, \ldots, k_{m}}\right)$. Then because $X_{d_{1}, d_{2}, \ldots, d_{m}}^{1, k_{2}, \ldots, k_{m}}=E_{d_{1}, d_{2}, \ldots, d_{m}}^{1, k_{2}, \ldots, k_{m}}-$ $\bigcup_{i=2}^{m} E_{d_{1}, d_{2}, \ldots, d_{i}, \ldots, d_{m}}^{1, k_{2}, \ldots, k_{i}+1, \ldots, k_{m}}$, it follows from 5 -lemma that we see that (as in [11]) $j_{1}{ }^{*}$ : $H_{c}^{j}\left(E_{d_{1}, d_{2}, \ldots, d_{m}}^{1, k_{2}, \ldots, k_{m}}\right) \rightarrow H_{c}^{j+2}\left(E_{d_{1}+1, d_{2}, \ldots, d_{m}}^{1, k_{2}, \ldots, k_{m}}\right)$ is bijective when $j>2 d-N\left(d_{1}, n\right)$ and surjective when $j=2 d-N\left(d_{1}, n\right)$. Then using Poincaré duality isomorphism, $j_{1_{*}}: H_{i}\left(E_{d_{1}, d_{2}, \ldots, d_{m}}^{1, k_{2}, \ldots, k_{m}} ; L\right) \rightarrow H_{i}\left(E_{d_{1}+1, d_{2}, \ldots, d_{m}}^{1, k_{2}, \ldots, k_{m}} ; L\right)$ is bijective when $i<N\left(d_{1}, n\right)$ and surjective when $i=N\left(d_{1}, n\right)$. Hence $j_{1}: E_{d_{1}, d_{2}, \ldots, d_{m}}^{1, k_{2}, \ldots, k_{m}} \rightarrow E_{d_{1}+1, d_{2}, \ldots, d_{m}}^{1, k_{2}, \ldots, k_{m}}$ is acyclic up to dimension $N\left(d_{1}, n\right)$ and this completes the proof of $\left(\dagger_{k}\right)$.

\section{§4. Generalizations}

Let $I$ be a collections of subsets of $\{1,2, \ldots, m\}$ such that $\operatorname{card}(\Lambda) \geq 2$ for any $\Lambda \in I$. We denote by $E_{d_{1}, \ldots, d_{m}}^{I, n}(X)$ the space

$$
E_{d_{1}, \ldots, d_{m}}^{I, n}(X)=\left\{\left(\xi_{1}, \ldots, \xi_{m}\right): \cap_{i \in \Lambda} \xi_{i}=\emptyset \text { for any } \Lambda \in I, \xi_{i} \in \mathrm{SP}_{n}^{d_{i}}(X)\right\}
$$

If $d_{1}=\cdots=d_{m}=d$, we write $E_{d}^{I, n}(X)=E_{d_{1}, \ldots, d_{m}}^{I, n}(X)$. If $X=\mathbb{C}$, we also write $E_{d_{1}, \ldots, d_{m}}^{I, n}=E_{d_{1}, \ldots, d_{m}}^{I, n}(\mathbb{C})$.

Theorem 4.1. If $m, n \geq 2, s_{d}: \operatorname{Hol}_{d}^{n}\left(S^{2}, X_{I}\right) \rightarrow \operatorname{Hol}_{d+1}^{n}\left(S^{2}, X_{I}\right)$ is a homotopy equivalence up to dimension $D(d ; m, n)$ when $n \geq 3$ and a homology equivalence up to dimension $[d / 2]$ when $n=2$. Here the number $D(d ; m, n)$ is given in (1.3.1).

Proof of Theorem 1.4. The assertion follows from Corollary 2.1 and Theorem 4.1.

Proof of Theorem 4.1. If $n=2$, the above theorem easily follows from [[11];appendix] as in the proof of Theorem 3.3. So we consider the case $n \geq 3$. If $d<n, \operatorname{Hol}_{d}^{n}\left(S^{2}, X_{I}\right)=\operatorname{Hol}_{d}^{*}\left(S^{2}, X_{I}\right)$. So in this case, the assertion also easily follows from [[5]; Theorem 4.1]. So we assume that $d \geq n \geq 3$. Then the proof is reduced to show the following Theorem 4.2. So it remains to show:

Theorem 4.2. Let $m \geq 2,1 \leq k \leq m$, and $d_{1}, \ldots, d_{m} \geq 2$ are integers. Then if $d_{k} \geq n \geq 3$, the stabilization map $j_{k}: E_{d_{1}, \ldots, d_{k}, \ldots, d_{m}}^{I, n} \rightarrow$ $E_{d_{1}, \ldots, d_{k-1}, d_{k}+1, d_{k+1}, \ldots, d_{m}}^{I, n}$ is a homotopy equivalence up to dimension $N\left(d_{k}, n\right)$, where $N(d, n)=d+n-4$. 
Proof. It suffices to show the case $k=1$. The proof is similar to that of Theorem 3.3 and we shall sketch the main idea here.

Since $d_{1}, \ldots, d_{m} \geq 2$, the modified proof of Lemma 3.2 shows that the fundamental group of $E_{d_{1}, \ldots, d_{m}}^{I, n}$ is an abelian group. Hence it suffices to show that $j_{1}: E_{d_{1}, d_{2}, \ldots, d_{m}}^{I, n} \rightarrow E_{d_{1}+1, d_{2}, \ldots, d_{m}}^{I, n}$ is acyclic up to dimension $N\left(d_{1}, n\right)$. Let $L$ be a local coefficient system on $E_{d_{1}+1, d_{2}, \ldots, d_{m}}^{I, n}$ (and hence on any subspace). We denote by $I^{\prime}$ the set $I^{\prime}=\{\Lambda \cap\{1,2, \ldots, m-1\}: \Lambda \in I\}$ and let $E_{d_{2}, \ldots, d_{m}}^{I^{\prime}, n}(X)$ be the space defined by

$$
E_{d_{2}, \ldots, d_{m}}^{I^{\prime}, n}(X)=\left\{\left(\xi_{2}, \ldots, \xi_{m}\right): \cap_{i \in \Lambda} \xi_{i}=\emptyset \text { for any } \Lambda \in I^{\prime}, \xi_{i} \in \operatorname{SP}_{n}^{d_{i}}(X)\right\} .
$$

If we consider the projection $\pi: E_{d_{1}, d_{2}, \ldots, d_{m}}^{I, n} \rightarrow E_{d_{2}, \ldots, d_{m}}^{I^{\prime}, n}(\mathbb{C})$ given by

$$
\pi\left(\xi_{1}, \xi_{2}, \ldots, \xi_{m}\right)=\left(\xi_{2}, \ldots, \xi_{m}\right),
$$

we have a commutative diagram:

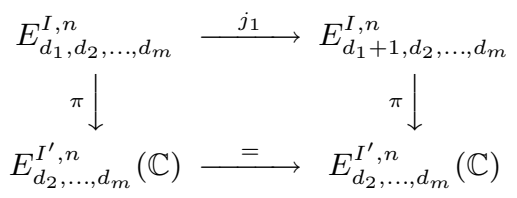

Let $E_{d_{1}, \ldots, d_{m}}^{k} \subset E_{d_{1}, \ldots, d_{m}}^{I, n}$ denote the subspace defined by

$E_{d_{1}, \ldots, d_{m}}^{k}=\left\{\left(\xi_{1}, \ldots, \xi_{m}\right) \in E_{d_{1}, \ldots, d_{m}}^{I, n}: \operatorname{card}\left(\bigcup_{1 \in \Lambda, \Lambda \in I}\left\{\cap \xi_{i}: 1 \neq i \in \Lambda\right\}\right) \geq k\right\}$.

Similarly, let $X_{d_{1}, \ldots, d_{m}}^{k} \subset E_{d_{1}, \ldots, d_{m}}^{I, n}$ and $Y_{d_{2}, \ldots, d_{m}}^{k} \subset E_{d_{2}, \ldots, d_{m}}^{I^{\prime}, n}(\mathbb{C})$ be subsets defined by

$$
\left\{\begin{array}{l}
X_{d_{1}, \ldots, d_{m}}^{k}=E_{d_{1}, \ldots, d_{m}}^{k}-E_{d_{1}, \ldots, d_{m}}^{k+1} \\
Y_{d_{2}, \ldots, d_{m}}^{k}=\pi\left(X_{d_{1}, \ldots, d_{m}}^{k}\right) .
\end{array}\right.
$$

Then the map $\pi$ restricts to a map $\pi: X_{d_{1}, \ldots, d_{m}}^{k} \rightarrow Y_{d_{2}, \ldots, d_{m}}^{k}$ and we have a commutative diagram

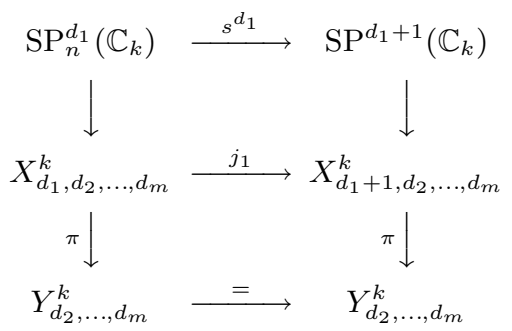

where vertical sequences are fibrations. 
Since $s^{d_{1}}$ is a homotopy equivalence up to dimension $N\left(d_{1}, n\right)$, the stabilization map $j_{1}: X_{d_{1}, d_{2}, \ldots, d_{m}}^{k} \rightarrow X_{d_{1}+1, d_{2}, \ldots, d_{m}}^{k}$ is also a homotopy equivalence up to dimension $N\left(d_{1}, n\right)$.

As in the proof of Theorem 3.3 we can use the Mayer-Vietoris exact sequence, 5-lemma, Poincaré duality and this to prove, by downwards induction on $k$, that the following statement holds:

$\left(\dagger_{k}\right) j_{1}: E_{d_{1}, d_{2}, \ldots, d_{m}}^{k} \rightarrow E_{d_{1}+1, d_{2}, \ldots, d_{m}}^{k}$ is acyclic up to dimension $N\left(d_{1}, n\right)$.

The statement of the theorem is given by $\left(\dagger_{1}\right)$.

\section{$\S 5 . \quad$ Proof of Theorem 3.1}

We need the following result.

Theorem 5.1. If $d \geq n \geq 3$ and $k \geq 1, s^{d}: \mathrm{SP}_{n}^{d}\left(\mathbb{C}_{k}\right) \rightarrow \operatorname{SP}_{n}^{d+1}\left(\mathbb{C}_{k}\right)$ is a acyclic up to dimension $N(d, n)=d+n-4$, where we take $\mathbb{C}_{k}=\mathbb{C}-$ $\{1,2, \ldots, k\}$.

Proof of Theorem 3.1. If $n=2$, the assertion follows from [[11]; appendix]. If $d<n$ and $n \geq 3$, then $\mathrm{SP}_{n}^{d}(X)=\mathrm{SP}^{d}(X)$. Hence the assertion follows from [[5]; Lemma 2.4]. So without loss of generalities, we may assume that $d \geq n \geq 3$. A similar proof of that of Lemma 3.2 shows that $\pi_{1}\left(\mathrm{SP}_{n}^{d}\left(\mathbb{C}_{k}\right)\right)$ is abelian. Hence the assertion follows from Lemma 3.1 and Theorem 5.1.

Proof of Theorem 5.1. Let $L$ be a local coefficient system on $\operatorname{SP}_{n}^{d+1}\left(\mathbb{C}_{k}\right)$. Since $\mathbb{C}_{k}$ is orientable, it extends to a local coefficient system on $\mathrm{SP}^{d}(\mathbb{C})$, which is denoted by the same letter $L$. We shall also use the same letter $L$ to denote its restriction to any (open or closed) subspace. Define the subspace $X_{n}^{d}(k) \subset$ $\mathrm{SP}^{d}(\mathbb{C})$ by $X_{n}^{d}(k)=\mathrm{SP}^{d}(\mathbb{C})-\mathrm{SP}_{n}^{d}\left(\mathbb{C}_{k}\right)$.

The stabilization map $s^{d}: \mathrm{SP}_{n}^{d}\left(\mathbb{C}_{k}\right) \rightarrow \mathrm{SP}_{n}^{d+1}\left(\mathbb{C}_{k}\right)$ naturally extends the map $s^{d}: \mathrm{SP}^{d}(\mathbb{C}) \rightarrow \mathrm{SP}^{d+1}(\mathbb{C})$. We also use the same letter $s^{d}$ to denote its restriction to any subspace. Remark that $s^{d}: X_{n}^{d}(k) \rightarrow X_{n}^{d+1}(k)$ naturally extends to the open embedding $s_{+}^{d}: X_{n}^{d}(k) \times \mathbb{C} \rightarrow X_{n}^{d+1}(k)$. This also induces the map $s_{+}^{d}: X_{n}^{d+1}(k)_{+} \rightarrow\left(X_{n}^{d}(k) \times \mathbb{C}\right)_{+}$, where $X_{+}$denotes the one-point compactification of a locally compact space $X$.

Because $\mathrm{SP}^{d}(\mathbb{C}) \cong \mathbb{C}^{d}$, it follows from the Alexander duality that for any $1 \leq i \leq 2 d-2$ there is an isomorphism $H_{i}\left(\mathrm{SP}_{n}^{d}\left(\mathbb{C}_{k}\right), L\right) \stackrel{\cong}{\rightrightarrows} H_{c}^{2 d-1-i}\left(X_{n}^{d}(k)\right)$ 
such that the following diagram is commutative:

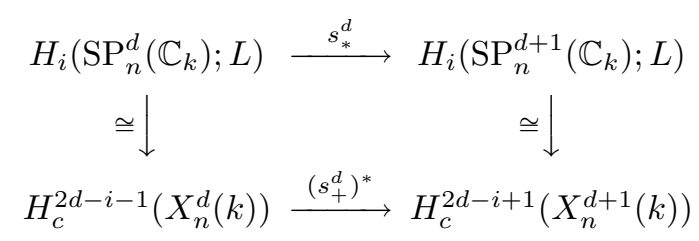

where $H_{c}^{k}(X)=H_{c}^{k}(X ; L)$ and $\left(s_{+}^{d}\right)^{*}$ denotes the composite of homomorphisms

$$
H_{c}^{k}\left(X_{n}^{d}(k)\right) \stackrel{\text { suspension iso. }}{\cong} H_{c}^{k+2}\left(X_{n}^{d}(k) \times \mathbb{C}\right) \stackrel{\left(s_{+}^{d}\right)^{*}}{\longrightarrow} H_{c}^{k+2}\left(X_{n}^{d+1}(k)\right) .
$$

Hence it suffices to show the following:

$(\dagger)\left(s_{+}^{d}\right)^{*}: H_{c}^{i}\left(X_{n}^{d}(k)\right) \rightarrow H_{c}^{i+2}\left(X_{n}^{d+1}(k)\right)$ is bijective when $i>M(d, n)$ and surjective when $i=M(d, n)$, where $M(d, n)=d-n+3$.

Define the subspaces $A_{n}^{d}(k)$ and $\Sigma_{n}^{d}(k)$ of $X_{n}^{d}(k)$ by

$$
A_{n}^{d}(k)=\bigcup_{i=1}^{k}\left(\{i\}+\mathrm{SP}^{d-1}(\mathbb{C})\right), \quad \Sigma_{n}^{d}(k)=X_{n}^{d}(k)-A_{n}^{d}(k) .
$$

Similarly, $s^{d}: A_{n}^{d}(k) \rightarrow A_{n}^{d+1}(k)$ and $s^{d}: \Sigma_{n}^{d}(k) \rightarrow \Sigma_{n}^{d+1}(k)$ naturally extend to the open embeddings $s^{d, 1}: A_{n}^{d}(k) \times \mathbb{C} \rightarrow A_{n}^{d+1}(k), s^{d, 2}: \Sigma_{n}^{d}(k) \times \mathbb{C} \rightarrow \Sigma_{n}^{d+1}(k)$. We note the following two results.

Proposition 5.1. $\quad\left(s_{+}^{d, 1}\right)^{*}: H_{c}^{i}\left(A_{n}^{d}(k)\right) \stackrel{\cong}{\rightrightarrows} H_{c}^{i+2}\left(A_{c}^{d+1}(k)\right)$ is an isomorphism for any $i \geq 1$.

Theorem 5.2. $\quad\left(s_{+}^{d, 2}\right)^{*}: H_{c}^{i}\left(\Sigma_{n}^{d}(k)\right) \rightarrow H_{c}^{i+2}\left(\Sigma_{c}^{d+1}(k)\right)$ is bijective when $i>M(d, n)$ and surjective when $i=M(d, n)$.

We postpone the proofs of the above two results and complete the proof of Theorem 3.1. Consider the commutative diagram

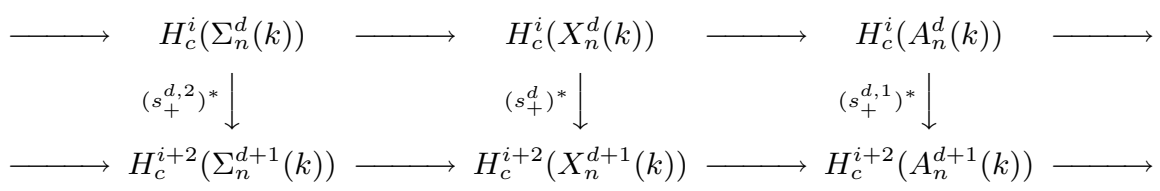

where horizontal sequences are exact. It follows from Proposition 5.1, Theorem 5.2 and 5-lemma that $\left(s_{+}^{d}\right)^{*}: H_{c}^{i}\left(X_{n}^{d}(k)\right) \rightarrow H_{c}^{i+2}\left(X_{n}^{d+1}(k)\right)$ is bijective when 
$i>M(d, n)$ and surjective when $i=M(d, n)$. This completes the proof of Theorem 3.1.

Proof of Proposition 5.1. We prove by induction on $k$. If $k=1, A_{n}^{d}(1) \cong$ $\mathbb{C}^{d-1}$ and the assertion trivial. If we assume that the assertion holds for $A_{n}^{d}(m)$ if $m<k$, then we can prove the assertion for $A_{n}^{d}(k)$ using Mayer-Vietoris exact sequence.

Proof of Theorem 5.2. From now on, we shall identify $\mathrm{SP}^{d}(\mathbb{C})$ with the space consisting of all monic polynomials $f(z) \in \mathbb{C}[z]$ of degree $d$. Then $\Sigma_{n}^{d}(k)$ may be regarded as the space consisting of all monic polynomials $f(z) \in \mathbb{C}[z]$ of degree $d$ such that $f(j) \neq 0$ for any $1 \leq j \leq k$ and that it has at least one root of multiplicity $\geq n$.

We write $N=[d / n]+1$ and let $\phi: \mathbb{C} \rightarrow \mathbb{C}^{N}$ be the Segre embedding given by $\phi(t)=\left(t, t^{2}, t^{3}, \ldots, t^{N}\right)$. Let $f(z)=g(z) \prod_{i=1}^{s}\left(z-\alpha_{i}\right)^{n} \in \Sigma_{n}^{d}(k)$ be any element, where

(i) $\alpha_{i} \in \mathbb{C}$ for each $1 \leq i \leq s$, and $\alpha_{i} \neq \alpha_{j}$ if $i \neq j$.

(ii) $g(z) \in \mathbb{C}[z]$ is a monic polynomial of degree $(d-n s)$.

Define the open $(s-1)$-dimensional simplex $\Delta\left(f ; \alpha_{1}, \ldots, \alpha_{s}\right)$ in $\mathbb{C}^{n}$ by

$$
\Delta\left(f ; \alpha_{1}, \ldots, \alpha_{s}\right)=\left\{\sum_{i=1}^{s} t_{i} \phi\left(\alpha_{i}\right): \sum_{i=1}^{s} t_{i}=1, t_{i}>0 \text { for each } i\right\} .
$$

We denote by $G\left(\Sigma_{n}^{d}(k)\right)$ the geometric resolution of $\Sigma_{n}^{d}(k)$ defined by

$$
G\left(\Sigma_{n}^{d}(k)\right)=\bigcup_{f \in \Sigma_{n}^{d}(k),\left\{\alpha_{i}\right\}} \Delta\left(f ; \alpha_{1}, \ldots, \alpha_{s}\right) \times\{f\} \subset \mathbb{C}^{n} \times \Sigma_{n}^{d}(k) .
$$

Let $q^{d}: G\left(\Sigma_{n}^{d}(k)\right) \rightarrow \Sigma_{n}^{d}(k)$ be the second projection. Then it is known [12] that $q^{d}: G\left(\Sigma_{n}^{d}(k)\right) \stackrel{\simeq}{\rightarrow} \Sigma_{n}^{d}(k)$ is a homotopy equivalence. It also extends to a homotopy equivalence $q_{+}^{d}: G\left(\Sigma_{n}^{d}(k)\right)_{+} \stackrel{\simeq}{\rightrightarrows} \Sigma_{n}^{d}(k)_{+}$, where $X_{+}=X \cup \infty$ denotes the one-point compactification of a locally compact space $X$. Let $F_{p}^{d} \subset$ $G\left(\Sigma_{n}^{d}(k)\right)_{+}$denote the subspace defined by

$$
F_{p}^{d}=\left(\bigcup_{s \leq p ; f \in \Sigma_{n}^{d}(k),\left\{\alpha_{i}\right\}} \Delta\left(f ; \alpha_{1}, \ldots, \alpha_{s}\right) \times\{f\}\right)_{+} .
$$

There is an increasing filtration

$$
\{\infty\}=F_{0}^{d} \subset F_{1}^{d} \subset \cdots \subset F_{[d / n]-1}^{d} \subset F_{[d / n]}^{d}=F_{[d / n]+1}^{d}=\cdots=G\left(\Sigma_{n}^{d}(k)\right)_{+},
$$


and if $1 \leq p \leq[d / n]$, there is a fibre bundle $q_{p}^{d}:\left(F_{p}^{d}-F_{p-1}^{d}\right) \rightarrow C_{p}\left(\mathbb{C}_{k}\right)$ with fibre $\mathbb{R}^{p-1} \times \mathrm{SP}^{d-n p}\left(\mathbb{C}_{k}\right)$. The open embedding $s^{d, 2}: \Sigma_{n}^{d}(k) \times \mathbb{C} \rightarrow \Sigma_{n}^{d+1}(k)$ induces a stabilization map $G\left(\Sigma_{n}^{d}(k)\right) \times \mathbb{C} \rightarrow G\left(\Sigma_{n}^{d+1}(k)\right)$ which preserves filtrations. In particular, if $1 \leq p \leq[d / n]$, there is a commutative diagram

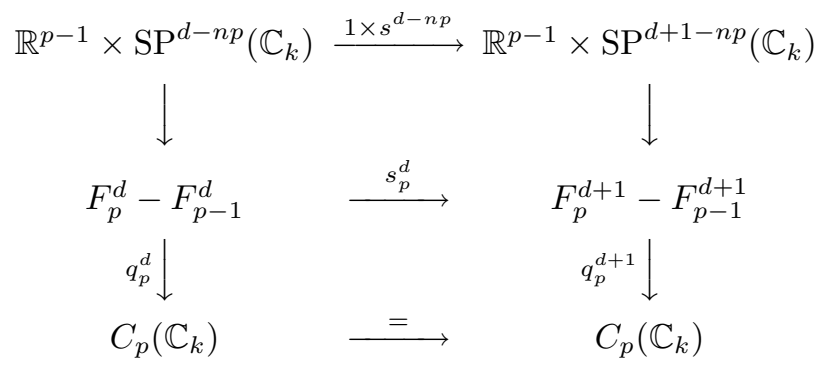

where vertical sequences are fibrations.

Since $s^{d-n p}: \mathrm{SP}^{d-n p}\left(\mathbb{C}_{k}\right) \rightarrow \mathrm{SP}^{d+1-n p}\left(\mathbb{C}_{k}\right)$ is a homotopy equivalence up to dimension $(d-n p)([5] ;(2.4)), s_{p}^{d}: F_{p}^{d}-F_{p-1}^{d} \rightarrow F_{p}^{d+1}-F_{p-1}^{d+1}$ is also a homotopy equivalence up to dimension $(d-n p)$. Because $q_{p}^{d}$ is a locally trivial, if $1 \leq p \leq[d / n] F_{p}^{d}-F_{p-1}^{d}$ is an orientable open manifold of dimension $(2 d-(2 n-3) p)$ and there is a commutative diagram

$$
\begin{array}{ccc}
H_{2 d-(2 n-3) p-i}\left(F_{p}^{d}-F_{p-1}^{d}\right) & \stackrel{\left(s_{p}^{d}\right)_{*}}{\longrightarrow} H_{2 d-(2 n-3) p-i}\left(F_{p}^{d+1}-F_{p-1}^{d+1}\right) \\
\text { P.d. } \downarrow \cong & \text { P.d. } \downarrow \cong \\
H_{c}^{i}\left(F_{p}^{d}-F_{p-1}^{d}\right) & \stackrel{\left(s_{p}^{d}\right)_{*}}{\longrightarrow} & H_{c}^{i+2}\left(F_{p}^{d+1}-F_{p-1}^{d+1}\right)
\end{array}
$$

Because $2 d-(2 n-3) p-i \leq d-n p$ if and only if $i \geq d-(n-3) p$, we obtain the following:

$\left(\dagger_{p}\right)$ If $1 \leq p \leq[d / n]$, then $\left(s_{p}^{d}\right)_{*}: H_{c}^{i}\left(F_{p}^{d}-F_{p-1}^{d}\right) \rightarrow H_{c}^{i+2}\left(F_{p}^{d+1}-F_{p-1}^{d+1}\right)$ is bijective when $i>d-(n-3) p$ and surjective when $i=d-(n-3) p$.

Consider the commutative diagram

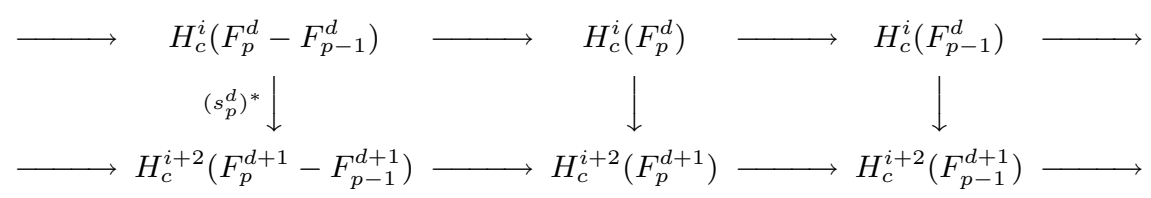

where horizontal sequences are exact. Then we have:

$\left(\ddagger_{p}\right)$ If $1 \leq p \leq[d / n]$, then $\left(s_{p}^{d}\right)_{*}: H_{c}^{i}\left(F_{p}^{d}\right) \rightarrow H_{c}^{i+2}\left(F_{p}^{d+1}\right)$ is bijective when $i>M(d, n)=d-n+3$ and surjective when $i=M(d, n)$. 
In fact, we can prove $\left(\ddagger_{p}\right)$ easily by induction on $p$. Since $F_{0}^{d}=\emptyset$, the case $p=1$ follows from $\left(\dagger_{1}\right)$. Next we assume that $\left(\ddagger_{p-1}\right)$ is true. Then $\left(\ddagger_{p}\right)$ follows from the above diagram and 5-lemma. Now we can prove Theorem 5.2 as follows.

First, assume $[d / n]=[(d+1) / n]=D$ and consider the commutative diagram

$$
\begin{aligned}
& H_{c}^{i}\left(\Sigma_{n}^{d}(k)\right) \stackrel{\left(q^{d}\right)^{*}}{\cong} \quad H_{c}^{i}\left(G\left(\Sigma_{n}^{d}(k)\right)\right) \quad \longrightarrow \quad H_{c}^{i}\left(F_{D}^{d}\right) \\
& \left(s_{+}^{d, 2}\right)^{*} \downarrow \quad\left(s_{D}^{d}\right)_{*} \downarrow \\
& H_{c}^{i+2}\left(\Sigma_{n}^{d+1}(k)\right) \stackrel{\left(q^{d+1}\right)^{*}}{\cong} H_{c}^{i+2}\left(G\left(\Sigma_{n}^{d+1}(k)\right)\right) \stackrel{=}{\longrightarrow} H_{c}^{i+2}\left(F_{D}^{d+1}\right)
\end{aligned}
$$

Then it follows from the above diagram and $\left(\ddagger_{D}\right)$ that the induced homomorphism $\left(s_{+}^{d, 2}\right)^{*}: H_{c}^{i}\left(\Sigma_{n}^{d}(k)\right) \rightarrow H_{c}^{i+2}\left(\Sigma_{c}^{d+1}(k)\right)$ is bijective when $i>M(d, n)$ and surjective when $i=M(d, n)$. Hence Theorem 5.2 is proved when $[d / n]=$ $[(d+1) / n]$.

Next, assume $D=[d / n]<[(d+1) / n]$. Consider the commutative diagram

$$
\begin{aligned}
& H_{c}^{i}\left(\Sigma_{n}^{d}(k)\right) \stackrel{\left(q^{d}\right)^{*}}{\cong} \quad H_{c}^{i}\left(G\left(\Sigma_{n}^{d}(k)\right)\right)=H_{c}^{i}\left(F_{D+1}^{d}\right) \quad \stackrel{=}{\longrightarrow} H_{c}^{i}\left(F_{D}^{d}\right) \\
& \left(s_{+}^{d, 2}\right)^{*} \downarrow \quad\left(s_{D}^{d}\right)_{*} \downarrow \\
& H_{c}^{i+2}\left(\Sigma_{n}^{d+1}(k)\right) \stackrel{\left(q^{d+1}\right)^{*}}{\cong} H_{c}^{i+2}\left(G\left(\Sigma_{n}^{d+1}(k)\right)\right)=H_{c}^{i+2}\left(F_{D+1}^{d+1}\right) \stackrel{j_{D}^{*}}{\longrightarrow} H_{c}^{i+2}\left(F_{D}^{d+1}\right)
\end{aligned}
$$

Now remark the following:

Lemma 5.1. If $i \geq 3 D+1, j_{D}^{*}: H_{c}^{i+2}\left(F_{D+1}^{d+1}\right) \cong H_{c}^{i+2}\left(F_{D}^{d+1}\right)$ is an isomorphism.

We postpone the proof of Lemma 5.1 and complete the proof of Theorem 5.2. Since $M(d, n) \geq 3 D+1$, it follows from the above commutative diagram, $\left(\ddagger_{D}\right)$ and Lemma 5.1 that $\left(s_{+}^{d, 2}\right)^{*}: H_{c}^{i}\left(\Sigma_{n}^{d}(k)\right) \rightarrow H_{c}^{i+2}\left(\Sigma_{c}^{d+1}(k)\right)$ is bijective when $i>M(d, n)$ and surjective when $i=M(d, n)$.

Proof of Lemma 5.1. Since there is a fibre bundle $F_{D+1}^{d+1}-F_{D}^{d+1} \rightarrow$ $C_{D+1}\left(\mathbb{C}_{k}\right)$ with fibre $\mathbb{R}^{D}$, using the Thom isomorphism and Poincaré duality, there is an isomorphism $H_{c}^{i+2}\left(F_{D+1}^{d+1}-F_{D}^{d+1}\right) \cong H_{c}^{i+2-D}\left(C_{D+1}\left(\mathbb{C}_{k}\right)\right) \cong$ $H_{3 D-i}\left(C_{D+1}\left(\mathbb{C}_{k}\right)\right)$.

Hence, if $i \geq 3 D+1, H_{3 D-i}\left(C_{D+1}\left(\mathbb{C}_{k}\right)\right)=0$ and $H_{c}^{i+2}\left(F_{D+1}^{d+1}-F_{D}^{d+1}\right)=$ $H_{c}^{i+3}\left(F_{D+1}^{d+1}-F_{D}^{d+1}\right)=0$. So the assertion follows from the cohomology exact sequence with compact supports of the pair $\left(F_{D+1}^{d+1}, F_{D}^{d+1}\right)$. 


\section{Acknowledgements}

The author is indebted to M. A. Guest and A. Kozlowski for numerous helpful conversations concerning the topology of labelled configuration spaces and related topics.

\section{References}

[1] Atiyah, M. F. and Jones, J. D. S., Topological aspects of Yang-Mills theory, Comm. Math. Phys., 59 (1978), 97-118.

[2] Bredon, G. E., Sheaf theory, Graduate Texts in Math., 170 (second edition), SpringerVerlag, 1997.

[3] Cohen, R. L., Jones, J. D. S. and Segal, G. B., Floer's infinite dimensional Morse theory and homotopy theory, Progr. Math., 133 (1995), 297-325.

[4] Stability for holomorphic spheres and Morse theory, Contemp. Math., 258 (2000), 87-106.

[5] Guest, M. A., Kozlowski, A. and Yamaguchi, K., The topology of spaces of coprime polynomials, Math. Z., 217 (1994), 435-446.

[6] S Spaces of polynomials with roots of bounded multiplicity, Fund. Math., 116 (1999), 93-117.

[7] Guest, M. A., Instantons, rational maps, and harmonic maps, Mathemática Contemprânea, 45 (1994), 57-75.

[8] The topology of the space of rational curves on a toric variety, Acta Math., 174 (1995), 119-145.

[9] Hausmann, J-C. and Husemoller, D., Acyclic maps, Enseign. Math., 25 (1979), 53-75.

[10] Kallel, S., Particle spaces on manifolds and generalized Poincaré dualities, Quart. J. Math., 52 (2001), 45-70.

[11] Segal, G. B., The topology of spaces of rational functions, Acta Math., 143 (1979), 39-72.

[12] Vassiliev, V. A., Complements of discriminants of smooth maps, topology and applications, Amer. Math. Soc., Translations of Math. Monographs 98, 1992 (revised edition 1994).

[13] Yamaguchi, K., Spaces of holomorphic maps with bounded multiplicity, Quart. J. Math., 52 (2001), 249-259. 